\title{
SOME FORMULAS RELATED TO DILOGARITHMS, THE ZETA FUNCTION AND THE ANDREWS-GORDON IDENTITIES
}

\section{BRUCE RICHMOND and GEORGE SZEKERES}

(Received 27 March 1979)

Communicated by A. J. van der Poorten

\begin{abstract}
It is shown that non-trivial relations between certain values of the dilogarithm function can be obtained through the asymptotic comparison of coefficients of the expressions which appear in the Rogers-Ramanujan and Andrews-Gordon identities.

1980 Mathematics subject classification (Amer. Math. Soc.): primary $10 \mathrm{~J}$ 20; secondary 33 A 70 , $10 \mathrm{H} 05$.
\end{abstract}

\section{Introduction}

The Rogers-Ramanujan identities have several interesting generalizations (see Andrews (1976), Chapters 7 and 8 for literature). Perhaps the most notable among these are the identities of Andrews and Gordon,

$$
\sum_{n_{1}, n_{2}, \ldots, n_{r}>0} \frac{q^{\Sigma_{i-1}^{r} N_{i}^{2}}}{\Pi_{i=1}^{r} \Pi_{v=1}^{n_{i}}\left(1-q^{v}\right)}=\prod_{n \neq 0, \pm(r+1) \bmod (2 r+3)}\left(1-q^{n}\right)^{-1}
$$

and the identities of Andrews,

$$
\sum_{n_{1}, n_{2}, \ldots, n_{r}>0} \frac{\Pi_{v=1}^{N_{r}}\left(1+q^{2 v-1}\right) q^{2 \Sigma_{i-1}^{r} N_{t}^{2}-N_{r}^{2}}}{\Pi_{i=1}^{r} \Pi_{v=1}^{n_{j}}\left(1-q^{2 v}\right)} \prod_{\substack{n \neq 2(\bmod 4) \\ n \neq 0, \pm(2 r+1)(\bmod 4 r+4)}}\left(1-q^{n}\right)^{-1}
$$

(Andrews (1976), Chapter 7, equations (7.3.7) and (7.4.7)), where $N_{i}=n_{1}+n_{2}$ $+\cdots+n_{i}$. 
Let $\sum_{k=0}^{\infty} a_{k} q^{k}$ be the power series expansion of either side of (1). Professor Andrews has (verbally) suggested to us that it might be worth while to examine the asymptotic behaviour of $a_{k}$ as $k \rightarrow \infty$ for the two sides of the identity and that non-trivial relations might result from the comparison. We shall find that this is indeed so and an estimate of the logarithm of the coefficients already supplies non-trivial relations between certain values of the dilogarithm function

$$
L i_{2}(x)=-\int_{0}^{x} \frac{\log (1-t)}{t} d t=\sum_{n=1}^{\infty} \frac{x^{n}}{n^{2}}
$$

(notation of Lewin (1958)). It is more convenient to express these relations in terms of the function

$$
L(x)=L i_{2}(x)+\frac{1}{2} \log x \cdot \log (1-x) .
$$

THEOREM 1. For given $r \geqslant 1$ let the sequence $\delta_{1}, \delta_{2}, \ldots, \delta_{r}$ be defined as

$$
\begin{aligned}
\delta_{1} & =2\left(1-\cos \frac{\pi}{2 r+3}\right), \\
\delta_{j+1} & =\delta_{j} / D_{j}^{2}, \quad j=1,2, \ldots, r-1,
\end{aligned}
$$

where

$$
D_{j}=\prod_{i=1}^{j}\left(1-\delta_{i}\right), \quad j=1, \ldots, r
$$

Then

$$
\sum_{i=1}^{r} L\left(\delta_{i}\right)=\frac{\pi^{2}}{3} \frac{r}{2 r+3}
$$

LEMMA. If $2 r+3$ is a prime $>5$ then the $\delta_{i}$ are units of the field of $\cos (\pi /(2 r+3))$.

We probably would not have proved this lemma had J. Lagarias not asked when the $\delta_{i}$ are units and had A. Odlyzko not computed the norms of the $\delta_{i}$ for $r<13$.

THEOREM 2. For given $r \geqslant 1$ let the sequence $\beta_{1}, \beta_{2}, \ldots, \beta_{r}$ be defined as

$$
\begin{aligned}
\beta_{1} & =2\left(1-\cos \frac{\pi}{2 r+2}\right), \\
\beta_{j+1} & =\beta_{j} / B_{j}^{2}, \quad j=1,2, \ldots, r-1,
\end{aligned}
$$

where

$$
B_{j}=\prod_{i=1}^{j}\left(1-\beta_{i}\right), \quad j=1, \ldots, r
$$


Then

$$
\sum_{i=1}^{r} L\left(\beta_{i}\right)=L\left(\frac{B_{r}}{1+B_{r}}\right)+\frac{\pi^{2}}{12}\left(\frac{2 r-1}{r+1}\right)
$$

Note that the numbers $\delta_{i}$ are all in the field of $\cos (\pi /(2 r+3))$ and the numbers $\beta_{i}$ in the field of $\cos (\pi /(2 r+2))$. For $r=1$ formula (5) gives the well-known $L((3-\sqrt{5}) / 2)=\pi^{2} / 15$, due to Landen 1760 , see Lewin (1958), p. 6. Formula (6) gives for $r=1$

$$
L(2-\sqrt{2})-L\left(\frac{2-\sqrt{2}}{2}\right)=\frac{\pi^{2}}{24}
$$

and for $r=2$

$$
L(2-\sqrt{3})+L\left(\frac{3-\sqrt{3}}{2}\right)-\frac{1}{2} L\left(\frac{\sqrt{3}}{2}\right)=\frac{\pi^{2}}{12} .
$$

Similar two-term formulae for values in the field of $\cos (\pi / 7)$ were obtained previously by Watson (1937). All can be obtained by suitable applications of the equation of Abel (1839), p. 251, formula (8),

$$
L(x)=\frac{1}{2} L\left(x^{2}\right)+L\left(\frac{x}{x+1}\right)
$$

and the trivial equation

$$
L(x)+L(1-x)=\frac{\pi^{2}}{6}
$$

both valid for $0 \leqslant x \leqslant 1$. It would be interesting to know whether (5) and (6) could be proved for all $r$ by suitable substitutions and applications of (7) and (8), without the help of asymptotic analysis and Rogers-Ramanujan type identities.

If in (5) we let $r \rightarrow \infty$ then the formula settles down to

$$
\sum_{k=2}^{\infty} L\left(\frac{1}{k^{2}}\right)=\frac{\pi^{2}}{6}
$$

which can also be derived easily from (4) and the trivial $L(1)=\pi^{2} / 6$. Similarly (6) gives, when $r \rightarrow \infty$,

$$
\sum_{k=1}^{\infty} L\left(\frac{1}{\left(k+\frac{1}{2}\right)^{2}}\right)=\frac{\pi^{2}}{3}-2 L\left(\frac{1}{3}\right) .
$$

Equation (9) is of particular interest because it can be rewritten with the help of expansion (3) of $\mathrm{Li}_{2}$ in the form

$$
\sum_{m=1}^{\infty} \frac{1}{m^{2}} \zeta(2 m)-\sum_{m=1}^{\infty} \frac{1}{m} \zeta^{\prime}(2 m)=\frac{\pi^{2}}{3},
$$


where $\zeta(s)=\sum_{n=1}^{\infty} 1 / n^{s}$ is the Riemann function. It shows that the sum of residues of the function

$$
\left(\frac{1}{z^{2}} \zeta(2 z)\right)^{\prime} \pi z \cot \pi z
$$

in the half-plane $\operatorname{Re} z \geqslant 0$ is zero. In particular (by partial integration)

$$
\int \zeta(2 z)\left(\frac{\cot \pi z}{z^{2}}-\frac{\pi}{z \sin ^{2} \pi z}\right) d z=0
$$

along any simple closed path surrounding the positive real axis (and excluding the negative integer places). This observation gives (by well-known estimates of the $\zeta$-function) the curious integral

$$
\int_{0}^{\infty} \frac{\operatorname{Im} \zeta(2 i t)}{t^{2}}\left(\frac{4 \pi t}{\left(e^{\pi t}-e^{-\pi t}\right)^{2}}-\frac{e^{\pi t}+e^{-\pi t}}{e^{\pi t}-e^{-\pi t}}\right) d t=\frac{\pi^{2}}{6}
$$

\section{Proof of the theorem}

We shall largely confine our attention to Theorem 1 . The proof of Theorem 2 is almost identical, with minor modifications which will be indicated in the text. Consider first the expansion of the left land side of identity (1)

$$
\sum_{n_{1}, \ldots, n_{r}>0} \frac{q^{\sum_{i-1}^{r} N_{i}^{2}}}{\Pi_{j=1}^{r} \Pi_{\nu=1}^{n_{j}}\left(1-q^{v}\right)}=\sum_{k=0}^{\infty} a_{k} q^{k}
$$

where $N_{i}=n_{1}+\cdots+n_{i}$. Then

$$
a_{k}=\sum_{n_{1}, \ldots, n_{r}>0} a(k ; \mathbf{n}), \quad \mathbf{n}=\left(n_{1}, \ldots, n_{r}\right),
$$

where

$$
a(k ; \mathbf{n})=\frac{1}{2 \pi i} \int \exp \left\{-\sum_{j=1}^{r} \sum_{\nu=1}^{n_{j}} \log \left(1-z^{\nu}\right)-\left(k-\sum_{j=1}^{r} N_{j}^{2}\right) \log z\right\} \frac{d z}{z}
$$

integrated along a suitable circle $z=e^{-\beta+i \theta},-\pi \leq \theta \leq \pi, \beta>0$. Hence

$$
\begin{aligned}
a(k ; \mathbf{n})=\frac{1}{2 \pi} \int_{-\pi}^{\pi} \exp \left\{-\sum_{j=1}^{r} \sum_{\nu=1}^{n_{j}} \log (1\right. & \left.-e^{-\nu \beta+\nu i \theta}\right) \\
& \left.+\left(k-\sum_{j=1}^{r} N_{j}^{2}\right)(\beta-i \theta)\right\} d \theta .
\end{aligned}
$$

Evaluation of the integral (14) runs on familiar lines (familiar from the asymptotic theory of partitions) and we follow the method in Szekeres (1953), hereafter referred to as PS. For details we shall lean heavily on this paper; in the 
case of a similar partition problem we have given more elaborate details in Richmond and Szekeres (1978). As we shall only be interested in the asymptotics of $\log a_{k}$, quite crude estimates will suffice.

First, determine the radius $e^{-\beta}$ of the circle of integration from

$$
\sum_{j=1}^{r} \sum_{\nu=1}^{n_{j}} \frac{\nu}{e^{\nu \beta}-1}=k-\sum_{j=1}^{r} N_{j}^{2}
$$

(the saddle point condition). Thus the radius depends not only on $k$ but also on $n_{1}, \ldots, n_{r}$. With trivial modifications of the method is PS we find, using the saddle point condition (15), that

$$
\log a(k ; \mathbf{n})=\left(k-\sum_{j=1}^{r} N_{j}^{2}\right) \beta-\sum_{j=1}^{r} \sum_{\nu=1}^{n_{j}} \log \left(1-e^{-\nu \beta}\right)+o(\sqrt{k}),
$$

where $\beta$ is determined from (15). The $o(\sqrt{k})$ term could be replaced by $O(\log k)$, but $o(\sqrt{k})$ suffices.

It is seen from (15) that $\beta$ is of order $1 / \sqrt{k}$, more precisely (by EulerMaclaurin summation)

$$
\frac{1}{\beta^{2}} \sum_{j=1}^{r} \int_{0}^{u_{j}} \frac{t}{e^{t}-1} d t=k-\frac{1}{\beta^{2}} \sum_{j=1}^{r} U_{j}^{2}+o(\sqrt{k}),
$$

where

$$
u_{j}=\beta n_{j}, \quad U_{j}=u_{1}+\cdots+u_{j}, \quad j=1, \ldots, r
$$

or

$$
k=\beta^{-2} \sum_{j=1}^{r}\left(\int_{0}^{u_{j}} \frac{t}{e^{t}-1} d t+\left(u_{1}+\cdots+u_{j}\right)^{2}\right)+o(\sqrt{k}) .
$$

From (15) and (16) and PS, p. 105

$$
\begin{aligned}
\log a(k ; \mathbf{n}) & =\sum_{j=1}^{r} \sum_{\nu=1}^{n_{j}}\left(\frac{\nu \beta}{e^{\nu \beta}-1}-\log \left(1-e^{-\nu \beta}\right)\right)+o(\sqrt{k}) \\
& =\frac{1}{\beta} \sum_{j=1}^{r}\left(2 \int_{0}^{u_{j}} \frac{t}{e^{t}-1} d t-u_{j} \log \left(1-e^{-u_{j}}\right)\right)+o(\sqrt{k}) .
\end{aligned}
$$

Hence $\log a(k ; \mathbf{n})$ is asymptotically equal to a constant times $\sqrt{k}$ where the constant depends of course on the $u_{j}$. We want to determine that system of $u_{j}$ for which the value of the constant is maximal.

One way would be to substitute $\beta^{-1}$ from (17) into the right hand side of (18) and to set all partial derivatives with respect to the $u_{j}$ equal to 0 . The resulting equations are involved and it is not easy to see how to solve them. It is more convenient to use finite differences instead of differentials. Following the 
method described in PS, Section 4, we calculate for fixed $j$ the difference

$\Delta \log a_{k}=\log a\left(k ; \mathbf{n}^{\prime}\right)-\log a(k ; \mathbf{n})$,

where

$$
\mathbf{n}^{\prime}=\left(n_{1}, \ldots, n_{j-1}, n_{j}+1, n_{j+1}, \ldots, n_{r}\right) .
$$

Let $\beta+\Delta \beta$ be the value of $\beta$ for the system $\mathbf{n}^{\prime}$. Then from (15)

$$
\begin{aligned}
& \sum_{\substack{i=1 \\
i \neq j}} \sum_{\nu=1}^{n_{i}} \frac{\nu}{e^{(\beta+\Delta \beta) \nu}-1}+\sum_{\nu=1}^{n_{j}+1} \frac{\nu}{e^{(\beta+\Delta \beta) \gamma}-1}=k-\sum_{i<j} N_{i}-\sum_{i>j}\left(N_{i}+1\right)^{2} \\
&=k-\sum_{i=1}^{r} N_{i}^{2}-2 \sum_{i>j} N_{i}-(r-j+1)
\end{aligned}
$$

and we obtain by lengthy but straightforward calculation, as in PS,

$$
\Delta \beta=\frac{\beta^{2}}{\sum_{i=1}^{r} \int_{0}^{u_{i}} \frac{t^{2} e^{t}}{\left(e^{t}-1\right)^{2}} d t}\left(\frac{u_{j}}{e^{u_{j}}-1}+2 \sum_{i>j} U_{i}\right)+o\left(\frac{1}{k}\right)
$$

and

$$
\begin{aligned}
\Delta\left(\sum_{i=1}^{r} \sum_{\nu=1}^{n_{i}}\right. & \left.\left(\frac{\nu \beta}{e^{\nu \beta}-1}-\log \left(1-e^{-\nu \beta}\right)\right)\right) \\
& =-\log \left(1-e^{-\mu_{j}}\right)-2 \sum_{i>j}\left(u_{1}+\cdots+u_{i}\right)+o(1) .
\end{aligned}
$$

Condition for $\log a(k ; \mathbf{n})$ to be maximal is that the expression (19) be 0 for every $j$, that is

$$
\log \left(1-e^{-u_{j}}\right)=-2 \sum_{i>j}\left(u_{1}+\cdots+u_{i}\right), \quad j=1, \ldots, r .
$$

To solve this system explicitly it is convenient to set

$$
u_{j}=\log \frac{1}{1-\delta_{j}}, \quad 0<\delta_{j}<1 .
$$

Then the equations to be solved are

$$
\log \delta_{j}=2 \sum_{i>j} \log D_{j}, \quad j=1, \ldots, r,
$$

where $D_{j}=\Pi_{i=1}^{j}\left(1-\delta_{i}\right)$, giving

$$
\delta_{1}=\prod_{i=1}^{r} D_{i}^{2}, \quad \delta_{j+1}=\delta_{j} / D_{j}^{2}, \quad j=1, \ldots, r-1 .
$$


We shall find later that these conditions determined $\delta_{1}$ and hence $\delta_{j}$ for $1 \leqslant j \leqslant r$ uniquely, and in fact

$$
\delta_{1}=2\left(1-\cos \frac{\pi}{2 r+3}\right) .
$$

Substituting (20) into (17) and (18) and noting that $\int_{0}^{\log (1 / 1-\delta)} t /\left(e^{t}-1\right) d t=$ $L i_{2}(\delta)$ we get, since there are at most $k^{r / 2}$ terms in (13) which contribute to $a_{k}$,

$$
\begin{aligned}
\log a_{k}= & \sqrt{k}\left(\sum_{i=1}^{r} L i_{2}\left(\delta_{i}\right)+\log ^{2} D_{i}\right)^{\frac{1}{2}} \\
& \cdot \sum_{i=1}^{r}\left(2 L i_{2}\left(\delta_{i}\right)+\log \delta_{i} \cdot \log \left(1-\delta_{i}\right)\right)+o(\sqrt{k}) .
\end{aligned}
$$

But

$$
\sum_{i=1}^{r} \log ^{2} D_{i}=\frac{1}{2} \sum_{i=1}^{r} \log \delta_{i} \log \left(1-\delta_{i}\right)
$$

since the right hand side is

$$
\sum_{j=1}^{r} \log \left(1-\delta_{j}\right) \cdot \sum_{i>j} \log D_{i}
$$

by (22) and this is clearly equal to $\Sigma_{i=1}^{r} \log ^{2} D_{i}$, the left hand side of (26). Hence we obtain from (4), (25) and (26)

$$
\log a_{k}=2 \sqrt{k}\left(\sum_{i=1}^{r} L\left(\delta_{i}\right)\right)^{\frac{1}{2}}+o(\sqrt{k})
$$

for the coefficient of expansion of the left hand side of identity (1).

The right hand side causes no problem. It is well known from the theory of partitions that if

$$
\prod_{n=1}^{\infty}\left(1-q^{n}\right)^{-1}=\sum_{k=0}^{\infty} p(k) q^{k}
$$

then $\log p(k)=\sqrt{2 / 3} \pi \sqrt{k}+o(\sqrt{k})$ and it is easy to verify that if $m$ out of the $(2 r+3)$ residue classes $\bmod (2 r+3)$ are omitted from the infinite product then for the coefficient fo expansion $a_{k}$ we get

$$
\log ^{2} a_{k}=\frac{2 r+3-m}{2 r+3} \cdot \frac{2}{3} \pi^{2} k+o(k) .
$$

In particular if $m=3$ then we get $\log ^{2} a_{k}=[2 r /(2 r+3)]\left(2 \pi^{2} / 3\right) k+o(k)$. This with (25) gives

$$
4 \sum_{i=1}^{r} L\left(\delta_{i}\right)=\frac{2 r}{2 r+3} \frac{2 \pi^{2}}{3}
$$


which is the statement of Theorem 1 . The proof of Theorem 2 goes in exactly the same manner except that in the saddle point condition (15) we now have $k-2 \Sigma_{j-1}^{r} N_{j}^{2}+N_{r}^{2}$ on the right hand side, therefore

$$
k=\frac{1}{2 \beta^{2}}\left\{\sum_{j=1}^{r} \int_{0}^{2 u_{j}} \frac{t}{e^{t}-1} d t+\int_{0}^{2 u_{r}} \frac{t}{e^{t}+1} d t+4 \sum_{j=1}^{r} U_{j}^{2}-2 U_{r}^{2}\right\}
$$

instead of (17).

Equations (20), expressing the condition of maximality, have to be replaced by

$$
-\log \left(1-e^{-u}\right)+\log \left(1+e^{-2 U_{r}}\right)=4 \sum_{i>j} U_{i}-2 U_{r}, \quad j=1, \ldots, r .
$$

Setting

$$
B_{j}=1-e^{-2 u_{j},} \quad U_{j}=\frac{1}{2} \log \frac{1}{1-\beta_{j}}, \quad 0<\beta_{j}<1,
$$

we obtain, as in (23)

$$
\beta_{1}=B_{1}^{2} B_{2}^{2} \cdots B_{r-1}^{2} B_{r}\left(B_{r}+1\right), \quad \beta_{j+1}=\beta_{j} / B_{j}^{2}, \quad B_{j}=\prod_{i=1}^{j}\left(1-\beta_{i}\right) .
$$

To complete the proof of Theorem 1 we still have to verify equation (24) and the corresponding equation for $\boldsymbol{\beta}_{1}$.

\section{Calculation of $\delta_{1}$ and $\beta_{1}$}

To show that the system (23) has exactly one solution with $0<\delta_{i}<1$, $i=1, \ldots, r$ and $\delta_{1}$ given by (24), we define the sequence of polynomials $\varphi_{m}(x)$, $m=0,1,2, \ldots$, by

$$
\begin{gathered}
\varphi_{0}(x)=1, \quad \varphi_{1}(x)=x, \\
\varphi_{m+1}(x)=(1+x) \varphi_{m}(x)-\varphi_{m-1}(x) \text { for } m>1
\end{gathered}
$$

and show: if we set $\delta_{1}=\delta, \varphi_{m}=\varphi_{m}(1-\delta), m>0$, then

$$
\delta_{m}=\delta / \varphi_{m-1}^{2} \text { for } m>1 \text {. }
$$

First we note that

$$
\varphi_{m-1}(x) \varphi_{m+1}(x)-\varphi_{m}^{2}(x)=x-1, \quad m>0,
$$

where for convenience we have set $\varphi_{-1}(x)=1$. For $m=0$ and $m=1$, equation (31) is obvious since $\varphi_{-1}(x)=1, \varphi_{0}(x)=1, \varphi_{1}(x)=x, \varphi_{2}(x)=x^{2}+x+1$, and for $m>1$ it follows by induction on $m$ since

$$
\begin{gathered}
\varphi_{m-1}(x) \varphi_{m+1}(x)=\varphi_{m-1}(x)\left((1+x) \varphi_{m}(x)-\varphi_{m-1}(x)\right), \\
\varphi_{m}^{2}(x)=\varphi_{m}(x)\left((1+x) \varphi_{m-1}(x)-\varphi_{m-2}(x)\right),
\end{gathered}
$$


hence

$$
\varphi_{m-1}(x) \varphi_{m+1}(x)-\varphi_{m}^{2}(x)=\varphi_{m-2}(x) \varphi_{m}(x)-\varphi_{m-1}^{2}(x)=x-1 .
$$

From (31) it is seen that

$$
\varphi_{m-2} \varphi_{m}-\varphi_{m-1}^{2}+\delta=0, \quad m \geqslant 1 .
$$

Now suppose (30) is true for some $m>1$. Then

$$
1-\delta_{m}=\frac{\varphi_{m-2} \varphi_{m}}{\varphi_{m-1}^{2}}
$$

because of (32), hence

$$
D_{m}=\prod_{i=1}^{m}\left(1-\delta_{i}\right)^{2}=\prod_{i=1}^{m} \frac{\varphi_{i-2} \varphi_{i}}{\varphi_{i-1}^{2}}=\frac{\varphi_{m}}{\varphi_{m-1}} .
$$

But by (23)

$$
\delta_{m+1}=\frac{\delta_{m}}{D_{m}^{2}}=\frac{\delta}{\varphi_{m-1}^{2}} \frac{\varphi_{m-1}^{2}}{\varphi_{m}^{2}}=\frac{\delta}{\varphi_{m}^{2}}
$$

and therefore (30) is true for $m+1$ hence for all $m<r$.

Now from the first equation (23)

$$
\delta=\prod_{i=1}^{r} D_{i}^{2}=\varphi_{r}^{2}
$$

by (34). Hence $\varphi_{r-1} \varphi_{r+1}=0$ by (32). But $\varphi_{r-1} \neq 0$ as seen from (33) since $\delta_{r}$ is finite, therefore $\delta$ is a root of the equation

$$
\varphi_{r+1}(1-\delta)=0 .
$$

But equation (29) shows that $\varphi_{m}(x)$ is that polynomial of degree $m$ for which

$$
\varphi_{m}(2 \cos \theta-1)=\frac{\sin (m+1) \theta-\sin m \theta}{\sin \theta}
$$

(see, for example, M. Abramowitz and I. Stegun (1964), p. 782, Table 22.7). The roots of $\varphi_{m}(x)=0$ are therefore given by $x=2 \cos \theta-1$ where $\sin (m+1) \theta=$ $\sin m \theta$. These are the places

$$
\theta=\frac{(2 k+1)}{(2 m-1)}, \quad k=0,1, \ldots, m-1 .
$$

Hence the roots of $\varphi_{m}(x)$ are

$$
2 \cos \frac{(2 k+1) \pi}{2 m+1}-1, \quad k=0,1, \ldots, m-1 .
$$

Thus $1-\delta=2 \cos (((2 k+1) \pi) /(2 r+3))-1$ for some $k \geqslant 0$, and only the largest of these, namely $2 \cos (\pi /(2 r+3))-1$ is acceptable if the condition 
$0<\delta_{m}<1$ is to be satisfied for all $m<\pi$. Hence

$$
\delta=2\left(1-\cos \frac{\pi}{2 r+3}\right)
$$

as required. This completes the proof of Theorem 1 .

The calculation of $\beta_{1}$ in Theorem 2 is very similar except that $\beta=\beta_{1}$ now satisfies

$$
\beta=1-\varphi_{r} \varphi_{r-1}-\varphi_{r}^{2}=-\varphi_{r-1} \varphi_{r+1}+\beta-\varphi_{r} \varphi_{r-1}
$$

hence $\beta$ is a root of $\varphi_{r}(1-\beta)+\varphi_{r+1}(1-\beta)=0$.

We now establish the lemma of Section 1. In fact we prove that if $n>1$, $2 n+1$ prime and $\nu$ a root of $\varphi_{n}(x)=0$ then $\varphi_{n}(\nu)$ is a unit of the field of $\nu$ for $m<n$.

First we recall that the roots of $\varphi_{n}(\nu)=0$ are the numbers

$$
\nu_{n, k}=2 \cos \frac{(2 k+1) \pi}{2 n+1}-1, \quad k=0,1, \ldots, n-1,
$$

are obviously algebraic integers. If $2 n+1>3$ is prime then they are clearly conjugates and their norm is \pm 1 since

$$
\varphi_{m+1}(0)=\varphi_{m}(0)-\varphi_{m-1}(0) \text {. }
$$

Hence

$$
\begin{aligned}
\varphi_{m}(0) & =0 & & \text { if } m \equiv 1(\bmod 3), \\
\varphi_{m}(0) & =1 & & \text { if } m \equiv 0 \text { or } 5(\bmod 6), \\
& =-1 & & \text { if } m \equiv 2 \text { or } 3(\bmod 6) .
\end{aligned}
$$

Therefore $\nu$ is a unit and clearly $\varphi_{n}(\nu)$ is an integer of the field. Furthermore since

$$
\varphi_{m}(x)=\prod_{l=0}^{m-1}\left(x-\nu_{m, l}\right)
$$

we have that

$$
\varphi_{m}\left(\nu_{n, k}\right)=\prod_{l=0}^{m-1}\left(\nu_{n, k}-\nu_{m, l}\right) .
$$

But

$$
\begin{aligned}
\nu_{n, k}-\nu_{m, l} & =2\left(\cos \frac{2 k+1}{2 n+1} \pi-\cos \frac{2 l+1}{2 m+1} \pi\right) \\
& =2 \sin \frac{\pi}{2}\left(\frac{2 k+1}{2 n+1}+\frac{2 l+1}{2 m+1}\right) \cdot 2 \sin \frac{\pi}{2}\left(\frac{2 k+1}{2 n+1}-\frac{2 l+1}{2 m+1}\right)
\end{aligned}
$$


which is a product of units, hence $\varphi_{m}\left(\nu_{n, k}\right)$ is a unit. This is $=1$ if $(2 n+1$, $2 m+1)=1$, in particular when $2 n+1$ is prime and $0<m<n$. This proves the statement.

It now follows immediately that $\varphi_{m}(1-\delta)$ is a unit for $0<m<r$ and in particular from (30) that $\delta_{m}$ is a unit for $1 \leqslant m \leqslant r$ and this gives the lemma.

Finally, we wish to verify that when $r \rightarrow \infty$, equation (6) does indeed settle down to (9). Equations (29) and (35) imply

$$
(2-\delta) \varphi_{r}-\varphi_{r-1}=0, \quad \varphi_{r}=(2-\delta) \varphi_{r-1}-\varphi_{r-2},
$$

hence

and from (33)

$$
\varphi_{r-1}=(2-\delta) \varphi_{r}, \quad \varphi_{r-2}=\left(3-4 \delta+\delta^{2}\right) \varphi_{r}
$$

$$
1-\delta_{r}=\frac{3-4 \delta+\delta^{2}}{(2-\delta)^{2}}
$$

But when $r$ is large, $\delta \rightarrow 0$ and so $\lim _{r \rightarrow \infty} \delta_{r}=1-\frac{3}{4}=\frac{1}{4}$. Similarly from (32) (with $m=r+1$ ) $\varphi_{r}^{2}=\delta$ hence from (30) $\delta_{r+1}=1$ and from (23)

$$
\delta_{r-1}=\delta_{r} D_{r-1}^{2}=\delta_{r} \frac{\varphi_{r-1}^{2}}{\varphi_{r-2}^{2}} \sim \frac{4}{9} \delta_{r} \sim \frac{1}{9} .
$$

Generally from (23)

$$
\begin{gathered}
\delta_{j}=\left(\frac{\delta_{j+1}}{1-\delta_{j+1}}\right)^{2} \frac{1}{\delta_{j+2}} \\
\delta_{r-j}=\left(\frac{\delta_{r-j+1}}{1-\delta_{r-j+1}}\right)^{2} \frac{1}{\delta_{r-j+2}}, \quad j=2,3, \ldots, r .
\end{gathered}
$$

From here it follows immediately by induction on $j$ that for fixed $j$,

$$
\lim _{r \rightarrow \infty} \delta_{r-j}=\frac{1}{(j+2)^{2}} \text {. }
$$

Equation (9) follows at once from this and (5).

Although equation (9) can be derived more directly from Abel's equation, our proof shows that it is linked with the identity

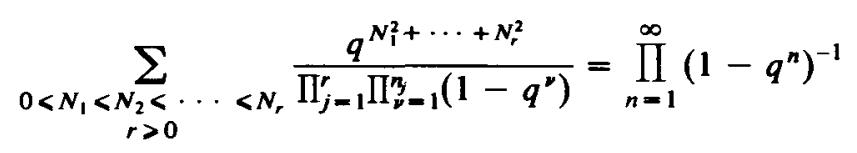

( $n_{j}=N_{j}-N_{j-1}, N_{0}=0$ ) which is obtained from (1) by letting $r \rightarrow \infty$. The right hand side is of course $\sum_{n=0}^{\infty} p(n) q^{n}$ where $p(n)$ is the number of partitions of $n$, 
and our derivation reveals an interesting link between the partition function $p(n)$ and (through (9) and (10)) properties of the $\zeta$-function.

\section{References}

N. H. Abel (1839), Oeuvres Complètes, vol. 2 (Gröndahl).

Milton Abramowitz and Irene A. Stegun (1964), Handbook of mathematical functions (National Bureau of Standards).

George E. Andrews (1976), The theory of partitions (Encyclopedia of Mathematics and its Applications, vol. 2, Addison-Wesley).

L. Lewin (1958), Dilogarithms (Macdonald, London).

B. Richmond and G. Szekeres (1978), The Taylor coefficients of certain infinite products', Acta Sci. Math. 40, 347-369.

G. Szekeres (1953), 'Some asymptotic formulae in the theory of partitions II', Quart. J. Math. Oxford Ser. 4, 96-111.

G. N. Watson (1937), 'A note on Spence's logarithmic transcendant', Quart J. Math. Oxford Ser. 8, 39-42.

University of Waterloo

Waterloo, Ontario

Canada N2L 3G1
University of New South Wales

Kensington, N.S.W. 2033

Australia 